of age. In Hasse's' series only 4 out of 995 began between 60 and 70.

"About 70 the disposition to epilepsy," says Dr. Maclagan, in his book on the Diseases of Advanced Life, "again increases in frequency;" and among various quotations of much in. terest, he says: "Within the short space of three years I had under my care no fewer than 10 cases, all occurring in men above 65 years of age, in a population of 500 , and in an institution from which epileptics are excluded when the disease is known prior to admission." Almost certainly senile epilepsy is not hereditary, or the attacks would have come on earlier in life, before the 20 th year. There have been cases wherein epileptic seizures have come on in infancy or childhood, have ceased, and recommenced at an advanced age, but these must be very fow and far between.

In Dr. Work Dodd's' series of 100 cases of epilepsy there were only 2 commencing in advanced life - that is, one at 57 and the second at 62 ; in neither was there any history of family predisposition. The nature of the seizures is precisely similar to those of earlier life; the two forms, the major and minor, occur, though the latter is probably the more frequent. The semi-comatose state after a fit of convulsions is frequently long, and sometimes deepens into the coma of death. Epilepsy, generally speaking, is not a fatal disease, except as just mentioned, when it comes on late on life, though even these persons do not die in the fits but in the after-state-the post-epileptic coma.

The diagnosis is easy after one attack of convulsions, though hysteria in women may simulate the complaint. The convulsions which are met with in the course of general paralysis of the insane might indeed cause some confusion, but the age of the patient, and the absence of any of the grand and vague imaginings and of any paralysis will sufficiently settle the question. Eclampsia or uræmic convulsions are more likely to be mistaken for epileptic seizures, but the character of the urine and the absence of symptoms of kidney disease will distinguish the cases. In all probability, cases of senile epilepsy have been mistaken for apoplexy. The suddenness of attack, the convulsions, and the after semi-comatose state might suggest the latter, but the absence of paralysis negatives it entirely.

Pathologically, there are several conditions such as cerebral hæmorrhage, thrombosis, embolism, and atheroma, which cause acute softening of the brain, but in all there will be paralysis. Again, in chronic progressive softening of the brain, which is most frequent between 60 and 80 , hemiplegia is constant and convulsions are very rare.

In epileptic children, especially where the brain lesion has been large, causing paralysis, the mental state may be actually that of an idiot. In adult epilepsy, sometimes not much mental failure is noted, though some impairment of memory is frequent. In the epilepsy of advanced life, the brain suffers very markedly after each fit of convulsions; the intellect may be perceptibly clouded, the semi-comatose state is less easily recovered from, more or less entire forgetfulness of the immediate past may come on, and the patient may live again in a world of forty years ago. The temper too often becomes feverish and irritable. In one of the cases, which will be given directly in some detail, the mental condition was fair till within a few weeks of his death; in the second case the mental condition has been much as I have just described.

Cerebral and cerebellar tumours again are frequently attended by fits of convulsions, but the vomiting, optic neuritis, and headache, as well as, frequently, paralysis, will prevent these cases being taken for epilepsy. Weakness in the legs indeed I have seen in epileptic cases almost approaching paralysis, but it soon went away when the bromide treatment was diminished.

As to the pathology of senile epilepsy little can be said, seeing that so little is certain about the pathology of idiopathic epilepsy. Convulsions are most frequently caused by cortical lesions, as in those occurring in cerebral tumour, etc.. but then there is almost always some paralysis consequent on the brain lesion. Also in this senile epilepsy we have to consider the gradual failure of the mind which depends so

Quain's Dict., Epilepsy. 5 Brain, 1893. largely on the exhaustion of the brain cells after each fit, whictlier major or minor.

Whether post-mortem investigation will reveal anything definite in these cases has yet to be seen. The treatment is the bromides of potassium and ammonium for the major attacks, and one or other of the sults of rinc for the minor ones. I also in both cases add some tincture of digitalis to the mixtures. The feeding and state of the bowels are care. fully attended to.

Case I K. T. was 73 years old when he had his first attack of convulsions, followed by coma for some hours. During this period, in which I saw him, it had much the appearance of apoplexy or uriemia. He thoroughly recovered. however, and had no more attacks for six years, on Aplil 17th, 1842. The next seizure was in July and August of that year; he was again seized on April 19th, 1893, became occasionally defirious, and finally sank in Mav of that year. II is attacks were well. marked major fits, and his mental state continued fairly good till he died at the respectable age of 78

CASE II. A. B. had uever had any symptoms of epilepsy till February 7 th, 1893, when she fell and cut herself on the sealp, and was unconscious for several hours. She had a few fits of convulsions thereafter, and her mental state perceptibly was weaker than before the fits. On May 12th she had another severe seizure; again on October $28 \mathrm{th}$, and on February she had another severe seizure; again on October $28 t h$, and on February ing fallen down in her bedroom. She sank into a comatose state and died ing fallen down

She was aged 73 also when the first fit occurred. I may mention that in neither case was there any ophthalmic change, the eyes indeed became weaker, as did all the other senses. In neither was there any paralysis. They presented typical examples, I think, of epilepsy occurring for the first time in old people, and for that reason they seemed to be worth while recording and criticising.

\section{A CASE OF SUBCLAVIAN ANEURYSM :}

Circulation controlled in the saC BY Digital pressure ON THE FIRS I PART OF THE VESSEL; MACEWEN'S OPERATION ; AMPUTATION AT THE BHOULDER-JOINT. ${ }^{1}$

By CHARLES A. MORTON, F.R.C.S.,

Surgeon to the Bristol General Hospital; Demonstrator of Anatomy in University College, Bristol.

Sir W. Ferausson proposed amputation at the shoulder-joint as a method of treating subclavian aneurysm, in the place of distal ligature alone, which was found unsatisfactory because branches came from the axillary artery between the ligature and the sac, which enlarged to supply collateral circulation to the upper limb. Fergusson therefore proposed to remove the upper limb, and thus do away with the tendency for these branches to enlarge. The first case so treated was Spence's celebrated case in 1864 . The aneurs $\mathbf{s m}$ diminished to one-third its former size, and the patient lived four years. In $1877 \mathrm{Mr}$. Holden ${ }^{2}$ amputated at the shoulder-joint for subclavian aneurysm, first tying the axillary artery nearer to the sac. At first the case promised well, but soon the aneurysm increased, and the man died. In $1880 \mathrm{Mr}$. Heath, at University College Hospital, treated a subclavian aneurysm in the same way ${ }^{3}$ and without benefit, the aneurysm finally consolidating from the introduction of needles, but the patient died of intercurrent disease. In Mr. Holden's case the aneurysm followed a strain to the shoulder in lifting a heavy weight, and in Mr. Heath's it was noticed a few weeks aftera fracture of the clavicle and first rib.

My case is, I believe, the fourth on record. The patient was in a desperate condition, large doses of morphine failed to relieve him, his mind was giving way, and he begged to have his arm off because of the great pain in it. Proximal digital pressure, direct pressure on the sac, and Macewen's method had all failed. He was greatly relieved of his pain after the amputation, probably by diminution of the tension on the cords of the brachial plexus, but the aneurysm increased in size, and he died in five weeks.

\section{History before Admission.}

J. S., aged 38, came to the Bristol General Hospital in Karch, 1893. He had been suffering pain in the left arm and above the left clavicle since the previous November, and Dr.

Read before the Bath and Bristol Branch of the British Medical Association, March, 1894.

2 St. Bartholomew's Hospital Reports, 1877.

3 BRITISH MBDICAI JOURNAL, 1880, vol, i, p. 205 
Parker detected a supraclavicular swelling. He remained under Dr. Parker's care as an out-patient until May 17th when he was admitted under Dr. skerritt, who transferred him to me on May $2 x$ th. During the eleven days he was in the medical ward he took 5 grains of iodide of potassium three times a day. During the time he was attending as a medical out-patient Dr. Parker did not think the aneurysm had been increasing in size, though the pain in his arm was getting worse. With rest in bed in the medical ward this decreased.

He was a strong muscular man, and had been a carrier of deal planks on the back of his left shoulder, where he had the subcutaneous thickening known as a "deal runner's pad." But for the last five years he had not been carrying deal planks, but heavy baskets of metal, which came down with a run on to his shoulder, and he often lifted these baskets with his left arm, so that there might have been considerable strain on the subclavian artery.

Condition of ANedrysi and UPPER Limb.

In the left supraclavicular region was a typical aneurysmal swelling, with loud rasping bruit and thrill. It was well defined, and about the size of a half section of a large orange. It extended outwards just to the anterior edge of the trapezius, internally to within an inch of the sternal end of the clavicle, downwards slight'y under the clavicle but not into into the axilla. Digital pressure behind the innermost inch of the clavicle almost stopped the pulsation in the aneurysm, and did not give decided pain. When the left arm lay in contact with the side there was no radial pulse, but it reappeared when the arm was abducted. The pain in the limb was most marked in the thumb and the radial side of the forearm just above it, and was accompanied by a numb feeling. He described the pain as like "burning fluid in the arm." There was no absolute anæsthesia anywhere, but sensibility was impaired over the inner and anterior part of the upper arm, the flexor surface of the forearm, and in all the fingers and thumb on both surfaces. but only beyond the joints between the first and second phalanges. The muscular grip of the hand was weak.

Digital Pressure on the First Part of the Subchavian.

On May 29th digital pressure was begun on this part of the artery with a 7 lbs. weight resting on the thumb. The arm was kept in the position in which the pulse was usually obliterated. Pressure was kept up for forty-eight hours, so as to completely control the pulsation in the aneurysm; it did not cause much pain. No consolidation of the aneurysm took place.

Use of Chloride of Calciom.

During the last twenty-four hours of this digital pressure 3 vi of calcium chloride were given (15 grs. every hour), and again 3 vj during the next twenty four hours. After this he had some smarting along the urethra and frequency of micturition, with alkaline urine and a little muco-pus, and the calcium chloride was stopped.

\section{MACewen's Treatuent.}

The aneurysm extended steadily and rapidly downwards below the clavicle, and the pain in the arm became very severe. The cords of the plexus could be felt stretched over it. He required frequent injections of morphine. On June lith a long, slender needle was inserted through the most prominent part of the aneurysm below the clavicle, and with it the opposite wall was repeatedly scratched at various spots. The needle became buried to the extent of 5 inches, 4 inches certainly lying between the anterior and posterior walls of the sac. The same needle was reinserted in two other places on the surface of the aneurysm below the clavicle, so as to scratch other areas of the posterior wall. It was impossible to reach the inside of the anterior wall where the aneurysm was so rapidly extending, as the needle could not be manipulated above the clavicle. The needles were in the sac for an hour and a-half, and were constantly moved from from one area to another. No pain was caused. During nearly the whole time digital pressure was kept up on the first part of the vessel. There was only oozing of a drop of blood as the needle was withdrawn, but this stopped at once with slight pressure. The needle and skin were, of course, thoroughly carbolised. The pulsation in the aneurysm seemed decidedly to lessen during the treatment, and at ono time seemed almost to have ceased-more so than when the treatment was discontinued.

On June 1:th there was decidedly less pulsation than before the needling, and it was hardly expansile at all. Above the clavicle very little pulsation of any kind could be felt. He required less morphine, and could use the hand and arm better, and it felt less numb. (On June 13th the condition was the same, except that just below the clavicle, above where the needle was inserted, the aneurysm was getting more prominent, and pulsated strongly with distinct expansile pulsation. After June 1:3th it continued to increase in this area, and our hopes of success from the needling were disappointed. By June 18th it was extending considerably towards the sternum, and presented high up in the axilla, and another needling was tried. This time the needle was inserted through the part of the aneurysm presenting high up in the axilla, and made to scratch the part rapidly extending below the clavicle. With the fingers on the outside I could feel the needle seratch it in various places about a dozen times. Once or twice it evidently stuck into it. It did not go easily through the wall in the axilla, seeming to indicate clotting here. Whilst needling pulsation most distinctly diminished in the part scratched, which before was the part in which it was most marked.

Direct Pressure on the Sac.

This was commenced directly after the second needling, by means of a 4 lbs. weight over a wool pad. For the first day it was kept on for five and a-half hours continuously, on the following day for two and a-half hours, and for two hours on the third day. After that he refused to continue the treatment longer, as it increased the pain in his arm. At an earlier period in the case it was not possible to try direct pressure on the sac, as the clavicle just bridged across it; it was only on the extension below the clavicle that direct pressure could be applied.

The pulsation in the aneurysm remained much less than before the second needling, but the bruit was louder and harsher, and the pain and odema (which had by this time come on) greater, the pain requiring hypodermic injections cf morphine every few hours.

On June 26th the axillary part of the aneurysm (which was the only part which pulsated as much as before the last needling) was scratched, and eight long slender needles were crossed in the sac for half an hour, as in Heath's case. The patient would not tolerate them for longer. No change took place in the aneurysm. The pain was extreme, even $\frac{1}{2} \cdot g r$. injections of morphine gave him little relief, and his mind seemed giving way.

Ampotation at Shodlder-Joint.

On June 27th I amputated at the shoulder-joint by Larrey's method. Digital pressure was made on the first part of the subclavian, and the axillary artery was followed by the fingers of my assistant, as the operation was completed, and compressed in the stump. It was partially obliterated. The pulsation in the aneurysm was absent for two days, perhaps because he remained considerably shocked after the operation. The pain was very greatly relieved. The aneurysm was much more prominent and extended nearer the sternum. This was probably due to its release from the upper limb, and the great relief from pain was probably due to diminished tension on the cords of the brachial plexus. By July 10th the wound was healed, but the aneurysm was getting larger; however, the pain was not nearly so severe as before the amputation. After that date the pain became more severe again, and the aneurysm continued to increase. On July 25 th and 26 th he had a rushing feeling in the scapular region. and the temperature rose to $103^{\circ}$, and ecchymosis appeared where the posterior axillary fold had been; and on August 4th he died exhausted.

\section{Post-Mortex Examination.}

The third part of the subclavian artery was lost in the aneurysm, which extended under the first and second parts of the vessel, pushing them up. The second part was adherent to the upper surface of the sac. The portion of the 


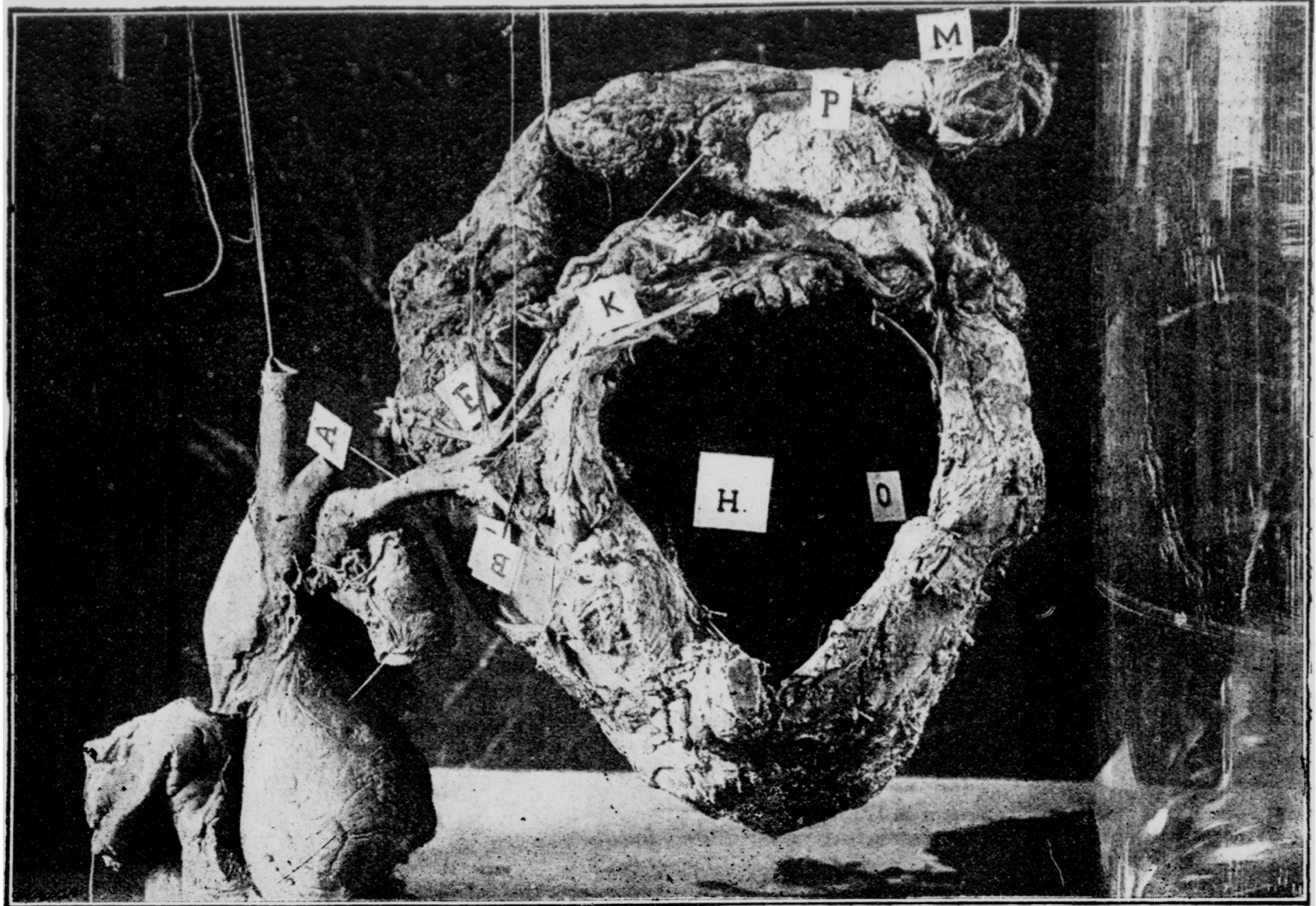

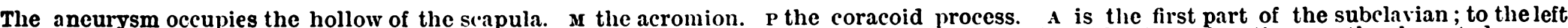
of it are seen the carotid and innominate arteries, the carotid just below $A$. $F$ is the thyruid axis, and $k$ the greatly elongated suprascapular artery. The pin on which the letter is is fixed is placed in the insertion of the scalenus anticus muscle, which is drawn upwards by cord. This rod can only in bey surface of the pleula and covering the first and second ribs.

aneurysm which extended upwards beneath the brachial plexus was about the size of a small orange. It contained laminated clot, half an inch thick, but mainly soft black clot. The cords of the plexus were slightly stretched over it, like the strings of a violin. The suprascapular artery was much elongated, but not obviously dilated. The first part of the subclavian artery was normal. The subclavian vein was obliterated about an inch before its termination. where it was adherent to the aneurysm. The phrenic nerve, vagus, carotid, and internal jugular were normal.

Below the clavicle the aneurysm was as large as an adult head, In what remained of the axilla, where the skin had become ecchymosed, the sac was formed by little else than skin, the discoloration being due to the blood in the sac soaking through. More posteriorly the collection of blood seemed like a secondary aneurysm communicating with the parent sac. The only part of the wall which resembled the interior of an artery was the anterior wall of the sac just below the opening of the artery into it, for a circular area of about $1 \frac{1}{2}$ inch in diameter. This closely resembled a thickened arterial wall with a smooth lining. There was no laminated clot on it, though it had been scratched by the needle inserted from the axilla. The continuation of the artery emerged from the upper surface of the aneurysm, a few inches from the opening of the second part of the vessel jnto it, so that the aneurysm extending downwards and backwards must soon have been formed only by the condensed surrounding tissues in this direction. The lateral branches of the intercostal nerves were found running through the more solid part of the clot. The great cavity of the aneurysm was filled with $3 \mathrm{lbs}$. of black soft clot, with layers of white clot here and there, but at the posterior aspect, where the aneurysm occupied the hollow of the scapula, there was a considerable thickness of laminated, partly white, but not very firm, clot. The first rib lay bare

in the sac. and was much eroded: the second rib was also eroded. and the aneurysm extended into the chest between them, but the parietal pleura was not perforated. The lung was slightly adherent at the margin of this area of bulging pleura, and between the two surfaces was a collection of slightly blood-stained serum, which was thus shut off from the general pleural cavity, but caused considerable collapse of the upper lobe of the lung. There was no laminated clot formed on the eroded first rib. The heart and other organs were normal.

\section{How Digital Pressure becane Possible on the} Proxinal Sine.

This case is, I imagine, probably the only one on record where it has been possible to apply digital pressure to the first part of the subclavian artery. It has been possible to do so in the case of an unusually high third part for aneurysm of the same part beyond, but probably never before to the first part. So extraordinary did it seem to me that we could actually press on the first part so as to control the circulation in the aneurysm that I thought it possible that the growing aneurysm had displaced the third part of the artery inwards and we really pressed on this, but I found that the pressure which controlled the circulation in the aneurysm could be applied as far inwards as the sternal attachment of the clavicle, and could not therefore be a displaced third part, and the post-mortem examination showed conclusively that it was the first portion of the artery pushed upwards by the aneurysm beneath, so that we really compressed the artery against the aneurysm.

\section{Question of Proxinat Ligatore.}

The question may be asked why did I not tie the artery on the proximal side of the aneurysm? Ligature of the first part of the right subclavian has been done fourteen times, 
and all the patients died of seconlary hamorrhage with the exception of two, who died before the usual period when this came on. So many branches come off from this part of the vessel that no satisfactory clot is formed. On the left side sir Astley ('ooper failed to secure the vessel, and is said to have wounded the thoracic duct. This deterred me from attempting ligature even with our modern absorbable ligatures on the method which aims at the non-division of the inner and middle coats.

Revarks on Macewhy's Method.

With regard to Macewen's treatment of aneurysm, I need only refer to his papers in the British Medical Jocresa for 1890 , and to two cases since published." His success, especially in one case of subclavian aneurysm, encouraged me to try it: and there is no doubt it considerably lessened the pulsation over the greater extent of the aneurysm, and promised well for a short time; but the rapidity with which the effect was produced seemed rather to indicate that the needles had simply acted as forrign bodies and produced some soft clot.

In connection with this method of treatment the following questions seem to me to arise. In an aneurysm of any size is not the wall always rough enough to start the formation of laminated clot without scratching? Is the exudation of leucocytes from the injury to the wall and their conversion into fibrin likely to produce more laminated clot than the deposition of leucocytes on the rough wall of the aneurysm:In Macewen's own cases the good result seems to have been far too gradual to have been due to clotting from the pre. sence of the needles acting as foreign bodies, but it is well to remember that there is always that element present in the treatment as well as the injury to the aneurysmal wall.

\section{ON THE MODE OF PERFORMING THE OPERATION OF VENTRO-FIXATION OF THE UTERUS, OR HYSTEROPEXY, IN CASES OF INTRACTABLE RETROFLEXION.}

BY JAMES BRAITHWAITE, M.D.LoNd.,

Obstetric Plysician and Surgeon to the Leeds General Infirmary.

As, in consequence of the interesting paper which was read by Drs. Leith Napier and F. F. Schacht at the Newcastle meeting of the British Medical Association' upon the subject which forms the title of this short paper, it is probable that the operation will be more extensively and more successfully performed than it had previously been, I venture to bring forward what is, I think, a further improvement in its mode of performance.

Dr. Napier's improvement was to pass several sutures, about four on an average, as low down on the anterior sur-

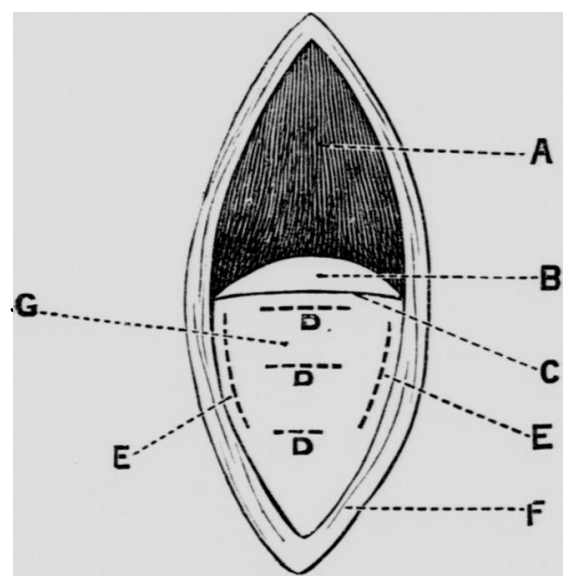

$\AA$, Interior of peritoneum ; $\mathrm{B}$, fundus uteri ; C, edge of uncut peritonenm; D, D, D, transverse sutures; E, E, longitudinal sutures; $F$, abdominal wall wound; (i, unopened portion of peritoueum geon from its outer surface.

Vol. ii, pp. 1107 and 1164 .

o Britieh Medical JourNal, 1s93, vol. i, p. 117 ; and Glaggov Med. Jour.,

1 British Medical JoUrNal, vol. ii, $19 ! 3$. face of the uterus as possible, so as to get the greater portion of it adherent to the abdominal wall. This makes the union firm and permanent, and renders it applicable to cases of intractable prolapse' as well as simply to retroflexion, which is a very important point. It also does away with the objection of Dr. Howard II. Kelly, of Baltimore, U.S., whose important article on the suhject appears in the Johns Hopkins Hospital lieports published recently, that the adhesion pro. duced clongates, so that soon the uterus is merely suspended by a band.

I have, since Dr. Napier's paper was read, done ten of these operations, with the following divergence from his plan. The central incision in the abdominal wall is made as usual low down, and with its lower end not more than an inch, or even less, above the pubes; but I have only cut through the peritoneum itself in the upper half of the incision ; in the lower half it is left intact, with as much fascia and cellular tissue as possible, the muscles however being drawn aside. Two fingers of the left hand are then passed in, and the uterus pulled up to, and held firmly in contact with, the uncut peritoneum exposed in the lower half of the wound. The fundus is then just visible above the edge of the peritoneum. The anterior surface of the uterus is now fastened to the peritoneum by silkworm gut sutures nearly as done by I)rs. Napier and Schacht.

This plan of operating seems to me to have several advantages. Two sutures can be placed from above downwards at the extreme edges of the anterior surface of the uterus, in addition to the usual transverse ones. This ensures a more complete and extensive coaptation of the peritoneum.

It must also, I think, be admitted that it is better to make a small opening into the peritoneum if it will do as well as a larger one, as the risk is thereby diminished, and that there is some risk is seen by Dr. Napier's cases.

The operation also as described is easier and more quickly done, in fact it only takes a few minutes to place the sutures when once the uterus is held up to the peritoneum. the proceedings being much simplified. It is also unnecessary to pass a temporary suture through the fundus, as recommended by Dr. Napier, in order to drag upwards and maintain in position the uterus whilst the permanent sutures are placed.

Lastly, I may ask what is the use of dividing, and so to a certain extent mutilating, the very part to which you are going to fix the uterus: There is no object gained by it.

$M y$ cases have done extremely well. but the success of the operation has been thoroughly established by Drs. Napier and schacht.

\section{A CASE OF CHLOROSIS AND AMENORRHCA WITH SYMPTOMS OF BRAIN DISEASE.}

\section{BY G. CRAWFORD THOMSON, M.B., II.R.C.S.,} London.

CASES of double optic neuritis with general brain symptoms and diplopia, ending in complete recovery without antisyphilitic treatment, are of sufficiently rare occurrence to justify the publication of the following instance :

Iriss 18:2. She was a cotal abstainer. No history of previous disease, 12uz. She with a especially with regard to acquired or herediary syphils or tuberculous disease, in herself or family. Her mother and mother's sister died of cancer. Menstruation commenced at the age of 16.1 , and was regular to 211; since then up to the day I saw her it had been very irregular and scanty, so'netimes stopping for as long as three months. Slie dates back her present illness to July, 1891, when she began to complain of general weakness, loss of appetite, headaches, giddiness, attacks of vomiting, and drowsiness. The headaches came on especially after moving, for instance after going upstairs, and sometimes kept her in bed all day, also at times preventing sleep at night. They were fre(fuently accompanied by severe romiting, which was independent of meals, and most often in the urning Attacks of faintness and giddiness were exceedingly frecuuent so that the patient iad to leave the class she was teaching ingly rreyent, so that on their account, seizing hold of anything within reach to prevent her falling; they even camc on in bed when turning from one side to the other. and mostly ended in vomit ng. Besides, she noticed that her sleep was more heavy and disturbed by bad dreams. On (ctcober $28 t h, 1891$, coming home for a walk, everything appeared double-for instance, on holding up one hand she saw two. The diplopia lasted with more or less severity for about four weeks.

Her eres were first examined on November 2:-1h, 1s91, by 1)r. Julius Jarobson, 10 whom I am indebted for the fillowing notes: Extreme pallor of iace, especially of conjunctivae and lips. Right eje vision 